\title{
Practical Research on Teaching Strategy of Information Technology Courses Based on Group Cooperative Learning
}

\author{
Yingxin Liu \\ Graduate school Shenyang University \\ Shenyang, China \\ yingxin888@126.com
}

\author{
Baoyin Liu \\ Graduate school Shenyang University \\ Shenyang, China \\ 1027803929@qq.com
}

\begin{abstract}
Group cooperative learning has been paid more and more attention by educational researchers, which has become an effective way for teachers to improve teaching effect. Information technology courses focus on improving students' information literacy, aiming at cultivating and improving students' ability of cooperation and communication. By using SPSS method to analyze students' learning results in one semester, the study finds that group cooperative learning teaching method can improve students' learning interest, skills, ability development and basic knowledge more than that of traditional teaching class, and the teaching effect is better. Teaching strategy of group cooperative learning has been verified effective and feasible in information technology courses.
\end{abstract}

Keywords-group cooperation; information technology: teaching strategies

\section{INTRODUCTION}

With continuous development of information technology education, the demand for talents has gradually changed from specialized talents with high knowledge reserve to compound talents with high quality. In "Outline of the National Medium and Long Term Education Reform and Development Plan (2010-2020)", it has been clearly pointed out that peopleoriented and comprehensive implementation of quality education should be strategic themes of educational reform and development[1], and "Outline of Basic Education Curriculum Reform (2001)" issued by the Ministry of Education also clearly points out that the important goal of curriculum reform is to cultivate students' ability of communication and cooperation[2]. The teaching method of group cooperation conforms to the requirement of "promoting students' ability of self-creation, unity and cooperation" in quality education. It can better improve students' ability of communication and cooperation, and make information technology class more efficient and meaningful.

\section{Teaching Strategy Model of Group Cooperative Learning}

According to theoretical basis of constructivism and teaching system design, a teaching strategy model suitable for group cooperation is designed. In the model, the main line is group cooperative learning, and teaching design is the key to the success of teacher group cooperative teaching. The model

2018 teaching reform research projects of undergraduate course of

common higher education in liaoning province. of group cooperative teaching strategy is shown in Figure 1.

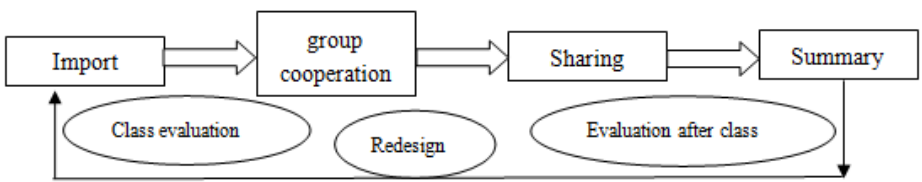

Fig. 1 Team Cooperative Teaching Strategy Model

\section{A. Design of Scenario Introduction Module}

In group cooperative learning, teachers first need to carefully design scenario introduction links, generally using wonderful video, stories and beautiful music and other content. Teaching works imported can be developed or used by teachers themselves. Teachers should choose the best resources on the Internet carefully according to the actual needs.

Teachers can design and develop materials of introduction link according to their needs, which will be more in line with the teaching content and teaching objectives, more in line with the characteristics of students' physical and mental development, and[3] stimulate students' interest in learning information technology. The effect of scenario introduction directly affects teaching effect. Considering the characteristics of pupils, scenario introduction should be short.

\section{B. Design of Task-driven and Group Cooperative Activity Module}

Teachers find problems in scenario introduction link, assign tasks to students, and show students the topics of classroom teaching after the introduction process. Teachers can appropriately design the difficulty of students' tasks according to the theory of "Nearest Development Zone", which is suitable for teaching objectives and contents, and conforms to the characteristics of students' physical and mental development. In the teaching design, tasks can not be set up in many ways to avoid students' boredom. Appropriate tasks can well stimulate students' curiosity.

Teachers first group the students into groups of 4-6 with a team leader. After the teacher assigns the task, the responsibility of the group members is divided. It is suggested that the group leader divide the task into several sub-tasks, let each member be responsible for a sub-task to explore, and 
finally group members are gathered together to explore. In group cooperative learning, teachers are assistants to help students guide details. Before each assignment, teachers should explain the rules of group cooperation and time limitation. The winning team of each task will get a bonus. Finally, the team counts the scores and divides the winners and losers.

The module is the key module in the whole teaching strategy, so teachers need to design it more carefully. In the process of group work, teachers should encourage students, mobilize students' participation in classroom activities, increase opportunities for participants to communicate with peers, expand students' thinking, and effectively guide group cooperative learning activities. Teachers should observe and evaluate the cooperation and performance of each group.

\section{Design of student sharing communication module}

After group cooperation and communication, the students complete the tasks assigned by the teachers. Each Group appoints a representative to report before the platform, share their works and successful experiences. When reporting and communicating, teachers can design forms of communication, such as small presenters, group competitions, debates and exhibitions, to encourage each group member to express positively, so that everyone has the opportunity to express themselves.

\section{Design of Teacher Summary Module}

Classroom content summary is freely expressed by students, guided by teachers, and written on the blackboard in the form of mind map to help students sort out the ideas of knowledge points and consolidate the content of classroom learning. Making students appropriately contact with mind map can cultivate students' logical thinking and develop good learning habits.

\section{E. Design of Teaching Evaluation Module}

The evaluation of group cooperative learning should not only focus on summative evaluation, but also on process evaluation. The diversified evaluation is accomplished by both students and teachers. The content of evaluation includes students' self-evaluation and teachers' evaluation. Teachers design self-assessment forms for students according to actual needs. In the table, five dimensions are designed for students' behavioral performance, and the group leader is responsible for the evaluation of each course. Teachers reflect on teaching according to students' self-evaluation and improve teaching design. Teachers' evaluation helps to increase students' learning information and the atmosphere of group cooperation by timely evaluation of students through classroom observation.

\section{RESEARCH PROCESS}

\section{A. Experimental subjects}

The precondition for teaching implementation of group cooperative learning is to fully understand the students. Through pre-teaching, observation of students and communication with the head teacher, students' personality, hobbies and learning ability can be mastered to lay a good foundation for the next group cooperative learning. The experiment was conducted in two classes with the same basic information and level, one of which was a group cooperative learning experimental class, the other was a traditional teaching control class.

\section{B. Course Practice}

The research chooses the curriculum of the third grade of primary school. The practice time of the course is one semester, and the total courses is 16 hours. One class adopts group cooperative teaching, while the other class still adopts traditional teaching. The teaching process of group cooperative learning classes mainly includes five links: scenario introduction, task-driven, group cooperation, students' sharing and communication, and teachers' summary. The traditional class teaching process is mainly based on Ausubel's pioneer teaching strategy, which includes presenting the pioneer organizer, presenting learning tasks and materials, and expanding and improving the cognitive structure. In practice, the evaluation methods include students' self-evaluation, mutual evaluation and teachers' classroom observation and evaluation. Teachers constantly improve their teaching design by summarizing the results of diversified evaluation.

\section{RESEARCH RESULTS}

After the text edit has been completed, the paper is ready for the template. Duplicate the template file by using the Save As command, and use the naming convention prescribed by your conference for the name of your paper. In this newly created file, highlight all of the contents and import your prepared text file. You are now ready to style your paper; use the scroll down window on the left of the MS Word Formatting toolbar.

\section{A. Design and implementation of questionnaires}

According to the evaluation criteria of teaching quality of primary and secondary school teachers in Chen Yukun's Educational Assessment Science and Richter's five-level scoring method, the design dimension, questioning method, option setting and number of options were adjusted and modified[4]. The reliability and validity of the questionnaire were analyzed, and experienced experts were consulted. After confirming the scientific nature of the questionnaire, a formal questionnaire survey was conducted. The Cronbach's reliability coefficient of the questionnaire is above 0.9, which shows that the results of the questionnaire are consistent and reliable. According to the results of KMO and spherical test, the KMO value is 0.89 , which is higher than 0.8 , which proves that it has good reliability. Therefore, the reliability and validity of the questionnaire is high, and the research data are highly scientific and authentic

According to the evaluation index of teaching quality in primary and secondary schools mentioned in Chen Yukun's Educational Evaluation, the questionnaire is designed from five dimensions: learning interest, ideological understanding, oneskill expertise, ability development and basic knowledge. Among them, 1-5 questions are learning interest, 6-10 questions are thinking and understanding, 11-15 questions are 
skills, and 16-23 questions are ability dimensions. The dimension of basic knowledge is tested in the form of final test papers.

After the completion of the questionnaire, 80 questionnaires were sent out, and 80 questionnaires were recovered. The recovery rate was $100 \%$. Among them, 80 valid questionnaires were valid and the validity rate was $100 \%$.

\section{B. Survey results}

1) Difference Analysis of Learning Interest, Ideological Recognition, Skills and Ability Development.

Through collecting questionnaires from group cooperative experimental classes and traditional teaching control classes, the results of the two classes in terms of learning interest, ideological understanding, one-skill skills and ability development are analyzed and statistics. The results of the experiment is $\mathrm{p}<0.05$. According to the statistical principle, the corresponding results of different classes have significant differences [5].

From the results, the $\mathrm{p}$ value of each dimension is less than 0.05 , which shows that there are significant differences between the experimental class and the control class in the results of the four dimensions. At the same time, according to the mean comparison, the score of the experimental group is significantly higher than that of the control group, which proves that the experimental class of cooperative learning in primary school improves the teaching effect than that of traditional teaching. The control class is more significant

\section{2) Difference Analysis of Basic Knowledge.}

The paper examines teaching effect of basic knowledge in the evaluation index of teaching quality in primary and secondary schools through the form of final test paper. The goal and requirement of the information technology curriculum standard in primary and secondary schools are student-centered attaching importance to basic knowledge, cultivating students' practical ability and innovative spirit. The design of the final test paper of information technology in the third grade of primary school refers to the requirements of new curriculum reform of information technology, follows the basic law of information technology teaching, and conforms to the middleaged stage of primary school students. Ability development level is helpful to improve students' information literacy and all-round development.

The final test paper is designed according to the teaching content of this semester. The purpose is to consolidate the practical operation with the basic knowledge, and make the theory and practice combine and complement each other and develop together. The test paper is divided into four parts, they are single choice questions, judgement right and wrong questions, filling in the blanks and connection questions. Before testing, consult relevant experts to determine the scientific and accuracy of the test paper.

After compiling the final test paper, the test was carried out for a primary school in Shenyang. A total of 80 students participated in the test. Finally, 80 papers were retrieved with a recovery rate of $100 \%$. Among them, 80 valid papers and $100 \%$ effective papers were obtained.
According to the statistical principle, the corresponding results of different classes have significant differences. From the results, there are significant differences between different classes in the results of different types of questions. At the same time, according to the mean comparison, the score of the experimental group is significantly higher than that of the control group.

There is a significant difference between the experimental class and the control class in the total score of the final test paper. The score of the experimental group is obviously higher than that of the control group. The experimental class of group cooperative learning has a better grasp of basic knowledge than that of the control class, which shows that group cooperative learning is one of the effective ways to improve students' basic knowledge.

\section{CONCLUSION}

Teachers use SPSS software to analyze the results of the questionnaire. The experimental class based on group cooperative learning has significantly improved the overall level of students' ideological understanding, learning interest, skills, ability development and basic knowledge. Teachers have better teaching effect in the five dimensions of small group cooperative classes, which is more in line with the requirements of students' all-round development.

Group cooperative learning takes students as the main body and meets the requirements of modern students' development. It has changed the disadvantages of traditional teaching with teachers as the main body, promoted students' all-round development, stimulated students' interest in learning information technology, fostered students' sense of unity and cooperation, and helped students improve their information literacy and scientific experience. It proves that the teaching strategy based on group cooperative learning can improve the teaching effect.

\section{REFERENCES}

[1] State Council of the People's Republic of China, "Outline of the National Medium and Long Term Education Reform and Developmen Plan (2010-2020) “. 2010

[2] Ministry of Education. Outline of Basic Education Curriculum Reform (Trial Implementation). 2001.

[3] National Information Curriculum Standards for Primary and Secondary Schools,. Beijing Normal University Press, 2011

[4] Chen Yuqi. "Education Evaluation" Beijing: People's Education Press, 1999, pp. 189-190.

[5] Zhang Jing, Shu Shihui, "Diversification of evaluation subjects of primary school mathematics classroom teaching under the new curriculum standard,”. Modernization of education, 2016,pp.347-348. 